\title{
Biométrie alaire de 3 espèces jumelles d'Anopheles maculipennis Meigen (Diptera : Culicidae) d'un gîte toulousain
}

\author{
J. Suzzoni-Blatger ${ }^{1}$ \\ J. Lauga ${ }^{2}$
}

Mots-clés : Anopheles maculipennis, espèces jumelles, biométrie alaire, analyse multidiscriminante.

L'étude de 7 paramètres alaires chez 3 espèces jumelles du «complexe maculipennis» : atroparvus, maculipennis et melanoon a été faite par l'analyse multidiscriminante. Elle montre que dans un gîte d'adultes de la région toulousaine, la longueur alaire est liée à l'espèce et peut servir de caractère discriminant : Anopheles atroparvus est un moustique à «ailes courtes», tandis que Anopheles maculipennis et Anopheles melanoon sont des moustiques à «ailes longues».

Wing biometry of three sibling species in the Anopheles «maculipennis complex»

Keywords : Anopheles maculipennis, sibling species, wing biometry, multidiscriminant analysis.

A study of 7 wing parameters in 3 sibling species of the «maculipennis complex» was carried out by multidiscriminant analysis. It shows that the wing length is correlated with species and can be used as a discriminant character.

Anopheles atroparvus is a short-winged mosquito, whereas Anopheles maculipennis (typicus) and A. melanoon are long-winged.

\section{Introduction}

Anopheles maculipennis est un complexe d'espèces jumelles dont la différenciation est basée sur des caractères morphologiques, biologiques et éthologiques aux divers stades de leur développement (De Buck et al. 1930, Hackett et al. 1935). Les plus couramment utilisés sont le chorion des œufs (Suzzoni-Blatger 1989, Guy et al. 1976) et la chétotaxie larvaire (Suzzoni-Blatger \& Sevin 1982).

La différenciation du complexe $A$. maculipennis a progressé ces dernières années grâce aux travaux de l'école italienne sur les systèmes gène-enzymes étudiés par les méthodes d'électrophorèse (Bianchi-Bullini et al. 1973 et 1980, Bullini \& Coluzzi 1978, Cianchi

1. Laboratoire de Parasitologie, Faculté de Médecine, 37, allées Jules-Guesde F - 31073 Toulouse Cedex.

E-mail : suzzoni@cict.fr

2. Laboratoire de Biologie quantitative, Université Paul-Sabatier, 118, route de Narbonne, F - 31062 Toulouse Cedex. et al., 1981, Suzzoni-Blatger et al. 1990). Certaines de ces espèces jumelles sont vectrices du paludisme, d'autres ne le sont pas. Il semblerait que nous assistions à une recrudescence des cas de paludisme autochtone dans le sud de la France pendant les étés chauds. Il serait pratique de pouvoir déterminer les adultes sur le terrain à l'aide d'un caractère morphologique simple, surtout lorsque coexistent plusieurs espèces jumelles dans les mêmes gîtes.

Un certain nombre de travaux concernant la longueur de l'aile chez diverses espèces de moustiques des genres Aedes et Culex ont été publiés (Van den Heuvel 1963, Haramis 1983, Nasci 1987 \& 1990, Siegel et al. 1994). Leurs résultats sont parfois contradictoires.

Chez Anopheles maculipennis, la longueur des ailes a longtemps été citée comme partiellement discriminante : les moustiques à «ailes courtes» étant considérés comme vecteurs du paludisme (Van Thiel 1927), les moustiques à «ailes longues» n'étant pas vecteurs (Swellengrebel et al. 1933, Hackett \& Missiroli 1935). 
De Buck (1926, in Van Thiel 1927) a montré une corrélation positive entre la longueur des ailes et la taille du corps. Il conclut que l'on peut prendre la longueur de l'aile comme mesure de la grandeur totale du corps de l'adulte. Van Thiel (1927) confirme ces travaux et conclut que la taille du moustique peut être jugée d'après celle de l'aile.

La différenciation des espèces jumelles d'un autre complexe de moustiques, le «complexe pipiens» a été effectuée par Makyia (1972) sur les organes sexuels du mâle et les ommatidies de la femelle en utilisant la fonction discriminante de Fisher.

Eritja (1996) utilise la biométrie de l'aile pour une analyse statistique discriminante en tant que technique de détermination du sexe chez Culex pipiens (Diptera, Culicidae) gynandromorphe.

Il nous a paru intéressant d'étudier la longueur des ailes des adultes du complexe maculipennis et de tester la validité de ce caractère par une étude biométrique. Nous avons utilisé la méthode d'analyse factorielle discriminante.

\section{Matériel et méthodes}

\subsection{Matériel}

150 femelles gorgées de sang ont été prélevées dans une bergerie des environs de Toulouse (Sud-Ouest de la France). Pour ne pas perturber l'équilibre du gîte, nous limitons volontairement les prélèvements d'adultes. Chaque femelle a été mise individuellement en élevage au laboratoire. Seules 31 femelles ont donné une descendance élevée jusqu'à la F1. Ce nombre de femelles ayant donné une descendance peut paraître faible ; il a plusieurs origines : les femelles gorgées sont très fragiles et peuvent souffrir pendant le transport, malgré toutes les précautions prises (aspirateur à bouche moins traumatisant que l'aspirateur électrique, glacière, etc.). Certaines femelles se noient sans pondre alors qu'elles sont gravides. D'autres femelles pondent, mais certains œufs n'éclosent pas ou bien il y a une mortalité plus ou moins importante des larves. La descendance de chaque femelle mère est très variable, pouvant aller d'un seul individu à 108 pour la F1.

Chaque ponte a été examinée et déterminée selon le critère de l'ornementation des œufs. Dans ce gîte, nous avons obtenu ainsi trois espèces jumelles appartenant au complexe maculipennis.

Les larves issues de chaque ponte sont élevées dans des conditions identiques d'alimentation (biscuits pour chien) de température et de densité larvaire (même nombre de larves dans un même volume d'eau par cristallisoir).
Au stade 4, la chétotaxie larvaire de chaque descendance a été examinée pour confirmation de la diagnose des œufs. Le premier jour de l'émergence, l'aile droite des adultes mâles et femelles est prélevée et montée à sec dans la gomme au chloral. Les mesures sont effectuées au micromètre après séchage à l'air libre pendant un mois.

De Buck et al. (1930) ont montré que la longueur de l'aile des adultes d'Anopheles maculipennis prélevés dans la nature se maintient identique dans leur descendance pendant deux générations. Les générations ultérieures n'ont pas été étudiées.

Boesiger (1978) a montré que la consanguinité n'a pas d'effet sur le poids des adultes du moustique Culex pipiens L. «jusqu'à la sixième ou même la dixième génération».

Nous avons donc choisi de mesurer les adultes de la F1 afin d'obtenir un échantillonnage suffisamment important pour être traité statistiquement. Nous avons essayé de sélectionner le plus petit nombre de caractères qui puisse aboutir à une séparation correcte des groupes.

Sept caractères ont été retenus : la longueur de l'aile du bout extrême de l'aile sans frange, jusqu'à la tangente à la base de l'aile, au niveau de la séparation de l'alula et de l'aile proprement dite ; la largeur de l'aile dans sa plus grande dimension : ces deux paramètres ont été proposés par Van Thiel (1927) car ils peuvent être mesurés de façon précise avec un micromètre oculaire.

Nous avons rajouté à ces mesures la longueur des trois côtés des triangles formés par les points d'intersection des trois nervures : $R_{2}-M_{2}-M C u$ (fig. 1). Ces mesures ont été choisies afin d'éliminer d'éventuelles déformations de l'aile dues à la dessication. Elles permettent l'évaluation des proportions de la structure de l'aile, c'est-à-dire de sa forme.

\subsection{Méthodes}

L'échantillonnage comprend la $1^{\text {ère }}$ génération issue des femelles mères récoltées soit 485 individus (244 mâles et 241 femelles).

L'étude des sept caractères alaires choisis représente un total de 3395 mesures.

Méthode de calcul :

La population globale a été séparée en fonction du nombre d'espèces jumelles et du sexe. Chacun des groupes a été projeté :

- sur l'axe de mesure des tailles (axe 1) 


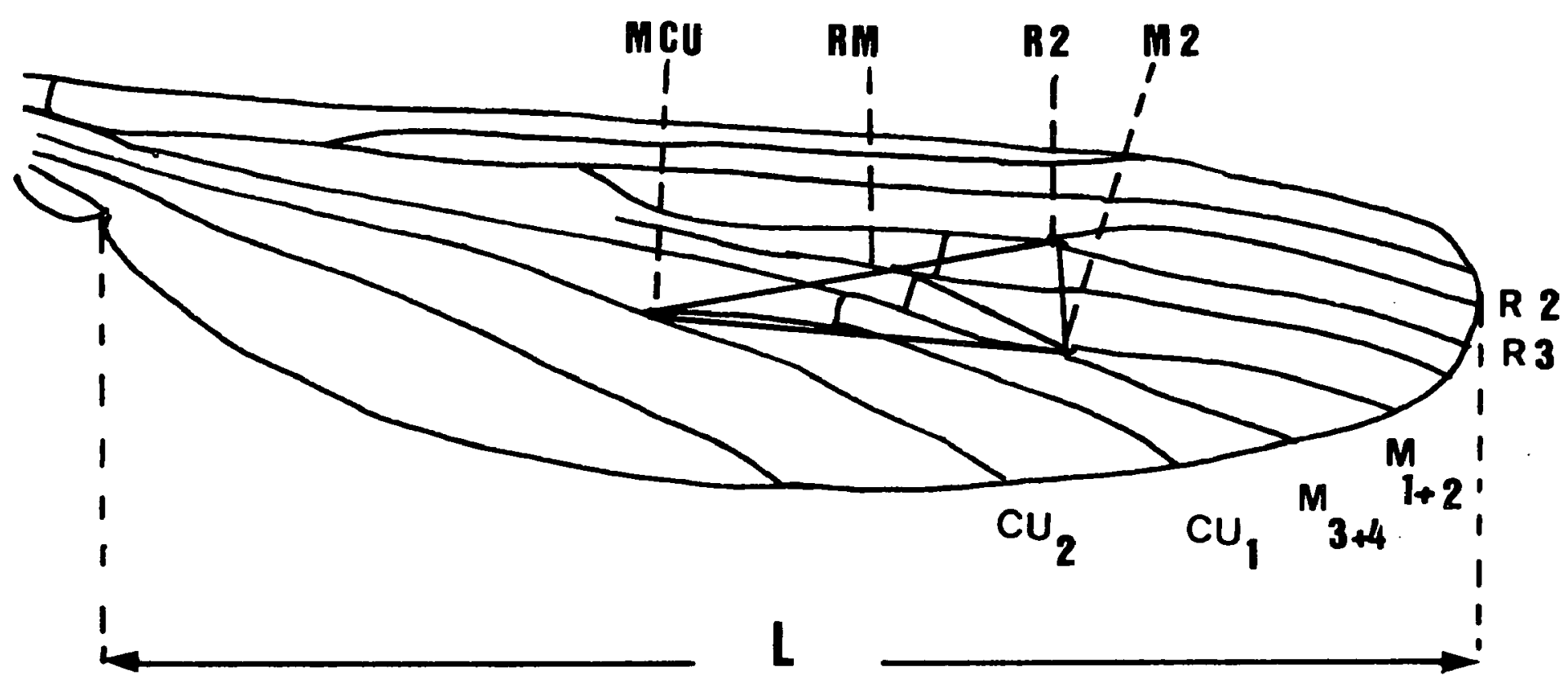

Fig. 1. Aile d'Anopheles.

Fig. 1. Anopheles wing.

- sur l'axe de mesure des différences de forme de l'aile (axe 2)

- sur la combinaison joignant dans le plan axe 1 / axe 2 les centres de gravité des groupes extrêmes observés. Les projections obtenues sont découpées en 50 classes distinctes pour l'axe 1 et l'axe 2 , et en 30 classes pour la combinaison axe $1 /$ axe 2 .

\section{Ajustements :}

Chacun des six groupes ainsi classé est comparé à une distribution normale. Cette opération vise à éprouver la normalité des distributions, condition indispensable pour les méthodes de calcul utilisées.

\section{Résultats}

Trois espèces jumelles ont été déterminées dans cette bergerie : Anopheles atroparvus, Anopheles maculipennis maculipennis (typicus) et Anopheles melanoon. 3.1. Comparaison des longueurs alaires selon les espèces

Le tableau $\mathrm{n}^{\circ} 1$ donne les moyennes des longueurs de l'aile droite des adultes de la F1, et le tableau $n^{\circ} 2$ les comparaisons statistiques.

Chez atroparvus qui est considéré comme un moustique microptère à «ailes courtes», la longueur des ailes apparaît significativement différente de celle de maculipennis (maculipennis). Celui-ci est un mous-

Tableau 1. Moyenne des longueurs de l'aile droite des adultes de la F1.

Table 1. Mean of the adult right wing lenght in the F1.

\begin{tabular}{|c|c|c|c|c|}
\hline Espèces jumelles & Sexe & $\begin{array}{l}\text { Moyenne des } \\
\text { longueurs en mm }\end{array}$ & $\begin{array}{l}\text { Nombre individus } \\
\text { mesurés }\end{array}$ & Ecart-type \\
\hline A. atroparvus & mâle & 3,58 & 124 & 0,247 \\
\hline $\begin{array}{l}\text { A. maculipennis } \\
\text { (typicus) }\end{array}$ & mâle & 4,11 & 105 & 0,177 \\
\hline A. melanoon & mâle & $.4,50$ & 15 & 0,192 \\
\hline A. atroparvus & femelle & 4,14 & 126 & 0,232 \\
\hline $\begin{array}{l}\text { A. maculipennis } \\
\text { (typicus) }\end{array}$ & femelle & 4,56 & 100 & 0,150 \\
\hline A. melanoon & femelle & 4,83 & 13 & 0,092 \\
\hline
\end{tabular}


Tableau 2. Comparaison des espèces jumelles par le test $t$ de Student

Tableau 2. Comparison of the sibling species with Studen $t$ test.

\begin{tabular}{|c|c|c|}
\hline Espèces & $\mathbf{t}$ de Student & Signification \\
\hline \multicolumn{3}{|l|}{ A. atroparvus } \\
\hline $\begin{array}{l}\text { A. maculipennis } \\
\text { (typicus) }\end{array}$ & 3,33 & +++ \\
\hline A. atroparvus & 2,71 & +++ \\
\hline \multicolumn{3}{|l|}{ A. melanoon } \\
\hline \multicolumn{3}{|l|}{ A. melanoon } \\
\hline $\begin{array}{l}\text { A. maculipennis } \\
\text { (typicus) }\end{array}$ & 2,20 & +++ \\
\hline
\end{tabular}

tique macroptère à «ailes longues». La valeur du $t$ de Student est de 3,33. La différence est très hautement significative. A. maculipennis (maculipennis) apparaît aussi significativement différent d'A. melanoon : $\mathrm{t}=$ 2,20. Pour Anopheles atroparvus, maculipennis (maculipennis) et melanoon de ce gîte toulousain, la longueur alaire est donc bien liée à l'espèce jumelle.

\subsection{Analyse multidiscriminante}

Les graphiques relatifs à l'axe 1 , à l'axe 2 et au discriminant sont représentés sexe par sexe sur une même feuille. On peut ainsi juger à l'œil de la qualité des résultats obtenus et de la variation du pouvoir discriminant selon l'axe factoriel auquel on s'adresse.

\subsubsection{Discrimination des populations}

L'analyse discriminante de départ fournissait un résultat quasi-complet dans le plan des deux premiers facteurs discriminants : 97,12\% d'inertie restituée. Des deux discriminants ainsi obtenus, le premier sépare les populations d'après leur taille $(89,12 \%$ d'inertie) et le deuxième d'après leur forme ( $8 \%$ d'inertie). Dans ces conditions, la taille des insectes constitue l'essentiel des différences observées (axe 1) et les différences de forme des ailes (axe 2), c'est-à-dire de proportions relatives entre les organes, ne contribuent que pour moins de $1 / 10^{\mathrm{e}}$ à la séparation morphologique des groupes. Il fallait toutefois vérifier si la combinaison des différences de taille et de forme, c'est-à-dire une combinaison linéaire axe 1 / axe 2 , ne permettrait pas d'obtenir un résultat plus contrasté.

\subsubsection{Normalité des distributions}

Les distributions obtenues ne peuvent, dans leur ensemble, être considérées comme normales. Les $\mathrm{X}^{2}$ d'ajustement le montrent et l'examen des graphiques le confirme. Le cas est spécialement prononcé pour melanoon en raison du trop faible effectif.

\subsubsection{Calcul des confusions}

Le calcul des confusions vise à apprécier dans quelle mesure les individus du groupe maculipennis (maculipennis) peuvent être correctement séparés des deux groupes extrêmes atroparvus et melanoon. Pour obtenir cette information, on commence par évaluer une grandeur, le D de Mahalanobis, assimilable à une «distance» entre les deux populations que l'on compare. La quantité $\mathrm{D} / 2$ est appelée écart-réduit de confusion. Elle équivaut à un écart-réduit gaussien et fournit directement un taux de confusion entre les deux échantillons. Pour la séparation atroparvus-maculipennis (maculipennis), la meilleure distinction s'observe sur l'axe 1 des tailles, suivi de l'axe 2 où la performance se dégrade. Les pourcentages de confusion sont :

$$
5,68 \% \text { (axe 1) .........24,49\% (axe 2) }
$$

Pour la séparation melanoon-maculipennis (maculipennis), les performances restent sensiblement identiques.

$$
37,67 \%(\text { axe } 1) \ldots \ldots . .30,48 \%(\text { axe } 2)\left(\text { tableau }^{\circ} 3\right) \text {. }
$$

\section{Discussion}

Cette analyse multidiscriminante montre que les adultes des espèces jumelles Anopheles atroparvus, maculipennis et melanoon d'une population du SudOuest de la France diffèrent par la longueur des ailes. Anopheles atroparvus est bien un moustique à «ailes courtes» et Anopheles maculipennis un moustique à «ailes longues».

L'analyse des différences de forme de l'aile, seule ou combinée avec la taille, n'améliore pas la discrimination des individus et le plus souvent la dégrade. L'espèce jumelle Anopheles melanoon possède trop peu d'individus pour que certains calculs puissent être conduits avec rigueur. Le reste de l'analyse n'apporte pas d'autre observation intéressante. 
Tableau 3. Chevauchement des groupes sur les axes 1 et 2 .

Table 3. Overlaping of the groups on the 1 and 2 axis.

Axe 1

Espèces comparées
D de

Mahalanobis
Ecart réduit

de confusion
Axe 2

Ecart réduit $\quad \%$ de de confusion confusion

\begin{tabular}{|c|c|c|c|c|c|c|}
\hline $\begin{array}{l}\text { A. atroparvus } \\
\text { A. melanoon }\end{array}$ & 2,60 & 1,30 & 9,71 & 2,46 & 1,23 & 10,92 \\
\hline $\begin{array}{l}\text { A.maculipennis } \\
\text { A. melanoon }\end{array}$ & 3,16 & 1,58 & 5,68 & 1,38 & 0,69 & 24,49 \\
\hline A. maculipennis & 0,63 & 0,31 & 37,67 & 1,02 & 0,51 & 30,48 \\
\hline
\end{tabular}

Cette analyse multidiscriminante confirme donc les travaux de Swellengrebel et al. (1926, in Hackett 1935). Ceux-ci ont montré que les différences des longueurs de l'aile étaient dues à des différences génotypiques et non pas aux conditions du milieu. Elle corrobore aussi les résultats d'Hackett et al. (1935) qui incluent la longueur des ailes comme critère d'identification de l'adulte.

Ces résultats confirment ceux de Siegel et al. (1994) montrant que des souches d'Aedes albopictus et d'Aedes triseriatus, d'origines géographiques différentes, ont une longueur de l'aile identique ; les résultats étant valables malgré la surpopulation et la privation de nourriture.

Les valeurs obtenues dans la région toulousaine n'ont pu être comparées statistiquement avec les valeurs de la bibliographie car celles-ci se révèlent le plus souvent incomplètes et donc inutilisables.

Cette différence morphologique chez l'adulte du complexe Anopheles maculipennis apparaît comme un critère supplémentaire en cas de difficulté de diagnose d'une souche, par exemple en cas d'importante hétérogénéité dans la chétotaxie larvaire ou dans la morphologie des œufs. Le rôle vecteur dans la transmission du paludisme varie selon les espèces jumelles du «complexe maculipennis» ce qui implique la nécessité d'une détermination précise.

\section{Travaux cités}

Bianchi-Bullini A. R., Cianchi R., Sabatini A., Coluzzi M. \& Bullini L. 1980. - Ricerche elettroforetiche su specie paleartiche del complesso Anopheles maculipennis (Diptera, Culicidæ). Atti XII Congr. Naz. Ital. Entomol., Roma, 2 : 255-259.

Boesiger B. 1978. - Comparaison de plusieurs caractères en fonction du degré de consanguinité chez Culex pipiens. Archiv. Genetik., $51:$ 193-216.
Bullini L. \& Coluzzi M. 1973. - Electrophoretic studies on geneenzyme systems in mosquitoes (Diptera-Culicidæ). Parassitologia, $15: 221-248$.

Bullini L. \& Coluzzi M. 1978. - Applied and theoretical significance of electrophoretic studies in mosquitoes (Diptera-Culicidæ), Parassitologia, $20: 7-21$.

Cianchi R., Sabatini A., Bullini C. \& Coluzzi M. 1981. - Differenziazione morfologica a genetica nei complesso Anopheles maculipennis e Anopheles claviger. Parassitologia, 23 : 158-163.

De Buck A., Schoute E. \& Swellengrebel N. H. 1930. - Racial differentiation of Anopheles maculipennis in Netherlands and its relation to malaria. Riv. malariol., 2 : 97-110.

Eritja R. 1996. - Wing biometry and statistical discriminant analysis as a technique to determine sex of a Culex pipiens (Diptera Culicidae) gynandromorph. J. econ. Entomol., $89:$ : 1338-1341.

Guy Y., Salières A. \& Boesiger E. 1976. — Contribution à l'étude du «complexe maculipennis» (Diptera-Culicidæ-Anophelinae). Mise au point en 1975. Ann. biol., $15: 227-282$.

Hackett L. W. 1935. - Les races d'Anopheles maculipennis. Riv. malariol., $14: 48-57$.

Hackett L. W. \& Missiroli A. 1935. - The varieties of Anopheles maculipennis and their relation to the distribution of malaria in Europe. Riv. malariol., $14:$ 45-109.

Haramis L. D. 1983. - Increased adult size correlated with parity in Aedes triseriatus. Mosq. News, 43 : 77-79.

Makyia K. 1972. - Dynamique des populations de moustiques dans le district de Nagoya. 4 - Examen morphologique du complexe d'adultes sauvages de Culex pipiens par utilisation d'une fonction discriminante. Jap. J. Sanit. Zool., 23 : 89-99.

Nasci R. S. 1987. - Adult body size and parity in field populations of the mosquitoes Anopheles crucians, Aedes toniorhynchus and Aedes sollicitans. J. amer. Mosq. Control Ass., 3 : 636-637.

Nasci R. S. 1990. - Relationship of wing lenght to adult dry weight in several mosquito species (Diptera Culicidæ). J. med. Entomol., 27 : 716-719.

Siegel J. P., Novak R. J. \& Ruesink W. G. 1994. - Relationship between wing length and dry weight of mosquitoes. J. amer. Mosq. Control Ass., 10 : 186-196.

Suzzoni-Blatger J. 1989. - Polymorphisme de l'ornementation des cufs chez Anopheles atroparvus (Diptera-Culicidæ). Annls Parasitol. hum. comp., $64:$ 77-80.

Suzzoni-Blatger J. \& Sevin A. 1982. - Etude de la chétotaxie larvaire du «complexe maculipennis» (Diptera-Culicidæ) dans la région toulousaine. Annls Parasitol. hum. comp., 57 : 649-654. 
Suzzoni-Blatger J., Cianchi R., Bullini L. \& Coluzzi M. 1990. «Le Complexe maculipennis» : critères morphologiques et enzymatiques de détermination. Annls Parasitol. hum. comp., 65 : 37-40.

Swellengrebel N. H. \& De Buck A. 1933. — Les races hollandaises de l'«Anopheles maculipennis» et leurs rapports avec les races italiennes. Bull. Soc. Path. exot., 26: 273-282.
Van Den Heuvel M. J. 1963. - The effect of rearing temperature on the wing lenght, thorax lenght, leg lenght and ovariole number of the adult mosquito Aedes aegypti (L.) Trans. roy. entomol. Soc. Lond., $115:$ 197-216.

Van Thiel P. H. 1927. - Sur l'origine des variations de taille de l'Anopheles maculipennis dans les Pays-Bas. Bull. Soc. Path. exot., $20: 366-390$. 